\title{
A Tutorial of Mobile Ad-Hoc Network (Manet) Routing Protocols
}

\author{
Palki Dhawan \\ ${ }^{1}$ (Department of Electronics and Communication, CGC, Gharuan)
}

\begin{abstract}
In mobile adhoc network nodes dynamically forms networks, there is no any central infrastructure, thus routing becomes a very important issue. Routing protocols used in mobile ad hoc networks (MANET) must adapt to frequent or continual changes of topology, while simultaneously limiting the impact of following these changes on wireless resources. This paper presents a comprehensive report and a comparative study of the different MANET routing protocols. The performance of such communication between nodes depends on various protocols. we have tendency to survey variety of the recent analysis results in routing space. In the following sections we have a tendency to present various existing routing protocols with their merits and demerits
\end{abstract}

Keywords: MANET, routing, network, communication

\section{Introduction}

A MANET is that the one consisting of distributed system of mobile nodes which might communicate with one another and nodes move independent of each other. [1]MANETs (Mobile Ad-hoc Networks) are one in all the fastest emerging networks. Nodes act as hosts as well as routers. Every node in a Manet is absolve to move severally in any direction, and can therefore change its links to the opposite devices often, each node must forward traffic unrelated to its own use, and so be a router. [2] The nodes in ad hoc networks are formed dynamically with no need for pre-configuration or an existing infrastructure like access point or base station [3]. MANETs usually present a high degree of mobility of nodes causing rapid changes in topology. [1] In several routing protocols specifically projected for MANETs, like AODV, OLSR, TORA, DYMO, every node broadcast messages periodically to maintain link status. In some cases neighbor table is used to notice the broken links, employed by topology based routing protocols like DSDV, AODV and DYMO.[4] The received hello messages are used to learn about every node's status [1]. MANETs are considered to be of good candidates due to many reasons: its simplicity for usage, robustness, speedy deployment and low cost. Its disadvantages comprise the complexity of routing due to the consistent move of nodes, mobility and dynamic topology, vulnerability of security due to the cooperation principle in MANETs, and the low computing power due to small devices used in MANETs.

The paper is divided into four sections. Section II explains the varied applications of the Manet. Section III in brief describes the varied routing protocols of Manet along with their benefits and Limitations and comparison table of all routing protocols. Finally, Section IV concludes the paper.

\section{Applications}

The application of Manet has become wide and varied from email to ftp to net services, because of their flexibility, MANETS are seen as important components in 4G design and ad hoc networking capabilities are believed to create a major a part of overall functionalities of next generation.

a. Personal area networking: Devices like mobile phones, laptops, PDAs create a network of less range to share data among each other in home/office wireless networking, conferences etc.

b. Military science networks or Military Environments: In military environments solider acts like nodes, as it's not possible to install base station in the enemy areas, hence it's very difficult for MANET to provide services in the areas. The application of MANET in the military services is coordination among the military soldiers and objects.

c. Industrial and Civilian Environments: Because of the easy deployment of MANET, it finds its use in many civilian activities like sports stadiums, military rooms, trade fairs, shopping malls, airports, e-commerce, business etc.

d. Emergency Operations: MANETs can be used in situations like disaster recovery, policing and fire fighting, crowd control etc. When conventional infrastructure based communication is damaged due to any calamities, MANETs can be used.

e. Education: In the field of education, MANETs can be used in universities, campus settings, and virtual classrooms and in ad hoc communications during meetings or lectures. 
f. Sensing element networks: In sensing element networks, MANETs can be used in data tracking of environmental conditions, animal movements and can also be used for home applications in smart sensors and actuators in consumer electronics.

g. Coverage extension: For the coverage extension MANETs used in linking up with the internet, intranets etc and extending cellular network access [5].

\section{Routing In Manet}

Routing algorithm determines the precise path of the route of communication, therefore the communication in the network depends on the potency and optimality of the routing algorithm. There are numerous routing obtainable in literature, according to its route finding types it's of two types; Proactive and Reactive [6]. Typically, nodes in Manet are characterized by their restricted power, restricted processing, restricted memory resources however high degree of quality. The wireless mobile nodes, in such networks, might dynamically enter in addition as leave the net-work. These nodes have restricted transmission vary and so, multiple hops are sometimes needed for message exchange among nodes in the network. For this reason, routing becomes a vital style issue of a Manet. This dynamic nature of mobile nodes will increase the routing quality in the network; hence routing is one of the important issues in Manet. In a Manet routing not solely rely on finding the trail quickly and expeditiously however conjointly it depends on many other factors together with choice of routers, topology, and location of request initiator. In Manet, routing area is that the most active research area [5].

MANET routing protocols can be mainly classified in two different categories based on their position information method and the routing information update. They are:

In this approach a mobile node uses its knowledge about recent connectivity of the network including the state of network links. [7] The routes are discovered and updated based on time, these routing protocol are classified into three categories:

(1) Proactive Routing Protocol

(2) Reactive Routing Protocol

(3) Hybrid Routing Protocol`

(1) Proactive Routing Protocol: Proactive protocols also known as "table driven" approach because information about the routes is maintained in tables. In this approach nodes, regularly discover path in the network to all nodes which are reachable and tries to keep consistent and up-to-date routing information in the routing table, These makes it easier for a source node to get a routing path immediately when required. [8] These routing tables are periodically exchange between nodes in network at set time interval. Some of the typical proactive routing protocols for MANET are Wireless Routing Protocol (WRP), Destination Sequence Distance Vector (DSDV) and Fisheye State Routing (FSR). In DSDV each nodes in the network regularly discover path to all nodes which are reachable and tries to keep consistent and up-to-date routing information in the routing table with the number of hops to reach each destination. Here the route entries are marked with a number known as sequence number to overcome problem known as loop problem. Routing tables are exchange periodically between nodes in network at set time interval in order to maintain table consistency. OLSR, an optimize version of DSDV. The key idea behind OLSR protocol is to reduce duplicate broadcast packets transmission in the same region. So if a node already received the message before it won't retransmit it again. WRP protocol uses four tables to maintain link cost, distance, routes, and message information retransmission. Here for each node, destination distance and last hop information are also included in route updates which are sent among neigh-boring, resulting in faster convergence.

\section{Advantages:}

(1) Lower route setup latency

(2) Proactive protocols can better address security vulnerabilities, because of the periodic exchange of control messages and routing table information.

(3) Modification of any route update can be overcome by the next scheduled update

\section{Disadvantages:}

(1) High routing overhead (periodic distribution of routing information).

(2) Stale routing information in highly dynamic topologies.

(2) Reactive Routing Protocol: In proactive protocol each node need to maintain a route to every other node at all times this is not required in reactive routing protocol. The main advantage of reactive routing protocol is that whenever a route is needed, it is available immediately. Previously the control overhead is costly because of frequently changes in link connectivity. Reactive routing approach is better because it does not continuously 
maintain a route between all pairs of network nodes. In this approach routes are only discovered when they are actually needed [9]. Some of typical Reactive routing protocols for MANET are Dynamic Source Routing (DSR)[10] protocol, Ad hoc On-demand Distance Vector (AODV) [11] protocol, and Temporally Ordered Routing Algorithm [12] (TORA). The DSR is a reactive routing approach, DSR uses source routing algorithm each data packet in DSR consists total routing information from source to destination. In each node in DSR a cache is maintained, which has route information from source to the destination. It includes two phases: Discovery of route and Route maintenance. AODV is the improvement of the DSDV protocol. AODV reduces the number of route broadcasts by not maintaining the complete list of routes as maintained by the DSDV algorithm, Here routes are available on on-demand basis. Like DSR, AODV also has two phase route discovery, and route maintenance. TORA is an on-demand source-initiated routing approach based on the link reversal concept Directed Acyclic Graph (ACG). TORA is loop-free and bandwidth- efficient routing protocol. Multiple routes from source to destination pair are provided by TORA. These features of TORA make it suitable for the environment which is highly dynamic or where the population of the node is dense. The only limitation of TORA comes from its dependency on synchronized clocks. This algorithm cannot be used if a node does not have an external time source or GPS positioning system.

\section{Advantages:}

(1) Reactive routing minimizes the dissemination of control packets in the network.

(2) Increases the available bandwidth for user data.

(3) Conserves the energy resources of mobile nodes.

(4) Reactive routing protocols introduce a medium to high latency.

\section{Disadvantages:}

(1) High routing overhead.

(2) Larger route set up latency

(3) Route discovery packet flooding

(3) Hybrid Routing Protocol: These protocols are the combination the proactive and reactive approaches that's why they known as hybrid routing protocol. Zone Routing Protocol (ZRP) is an example of such type of protocol is the. ZRP first partition the entire topology of the network into zones and then based on the strengths and weaknesses of routing protocols it apply different routing protocols between and within the zones. We can use any routing protocol between and within the zones. Here the size of each zone is decided by a parameter $r$ which describes the radius in hops. Since the proactive routing protocols keep an up to date view of the zone topology, that's why the Intra-zone routing means routing within zone is done by the proactive protocol, when communication takes place between nodes of the same zone these proactive routing protocol causes no initial delay. The routing between different zone routing is done by a reactive protocol. So the node does not require to keep the fresh state of the entire network.

\section{Advantages:}

(1) Reduces impact of disadvantages of proactive and reactive routing protocols.

(2) No route setup latency for short distance connections.

(3) Lower routing overhead due to reactive routing for further away destinations.

\section{Disadvantages:}

(1) More complex.

TABLE I

Comparison of different routing Protocols

\begin{tabular}{|c|c|c|c|}
\hline PROTOCOLS & PROACTIVE & REACTIVE & HYBRID \\
\hline Forwarding Method & $\begin{array}{c}\text { Wireless } \\
\text { Multihop } \\
\text { forwarding }\end{array}$ & $\begin{array}{c}\text { Wireless } \\
\text { Multihop } \\
\text { forwarding }\end{array}$ & $\begin{array}{c}\text { Wireless } \\
\text { Multihop } \\
\text { forwarding }\end{array}$ \\
\hline Recovery Strategy & $\begin{array}{c}\text { Multihop } \\
\text { forwarding }\end{array}$ & Carry \& Forward & both \\
\hline Realistic Traffic Flow & Yes & Yes & Yes \\
\hline Security & No & No & No \\
\hline Examples & DSDV, & DSR, & ZRP \\
& OLSR, & AODV, & \\
& WRP,FSR & TORA,DYMO & \\
\hline
\end{tabular}




\section{Conclusion}

In this paper, we present several routing protocols in MANET that may be a promising technology. We also presented some applications of MANET. The advantages and limitations of the studied protocols are also described. By learning totally different routing protocol in MANET we have seen that more performance analysis is needed to verify performance of a routing protocol with different routing protocols supported numerous situations. The table shows the comparative analysis of all the above stated routing protocols. The domain of Mobile Ad Hoc Network (MANET) and its related analysis are still in progression phases. The restricted practical deployable choices beneath completely different projects are strictly simulation based before their actual implementations.

\section{REFERENCES}

[1] Miguel Luis, Luis Bernardo, Rui Dinis, Paulo Pinto Rodolfo Oliveira, "The Impact of Node's Mobility on Link-detection based on Routing Hello Messages," in Wireless Communications and Networking Conference (WCNC), Sydney, NSW, 18-21 April 2010, pp. $1-6$.

[2] Ingrid Moerman, Bart Dhoedt and Piet Demeester Jeroen hoebeke, "An Overview of Mobile Ad Hoc Networks: Applications and Challenges," JOURNAL OF THE COMMUNICATIONS NETWORK, vol. 3, pp. 60-66, july 2004.

[3] M.B.,Al-Dubai, A.Y. and Buchanan, W. Khalaf, "A new adaptive broadcasting approach for mobile ad hoc networks," in Wireless Advanced (WiAD), 2010 6th Conference on, London, 27-29 June 2010, pp. 1 - 6.

[4] V.C, Singhal, M Giruka, "Hello protocols for ad-hoc networks: overhead and accuracy tradeoffs," in World of Wireless Mobile and Multimedia Networks, 2005. WoWMoM 2005. Sixth IEEE International Symposium on a, 13-16 June 2005, pp. 354 - 361.

[5] Aashish Choudhary, "Modified Adhoc on Demand Routing Protocol in Mobile Ad hoc Network," National Institute of Technology , Rourkela, Odisha, 769008, India, Thesis in Master of Technology July,2013.

[6] Petteri Kuosmanen, "Classification of ad hoc routing protocols," 2002.

[7] Humayun Bakht, "Survey of Routing Protocols for Mobile Ad-hoc Network," International Journal of Information and Communication Technology, 2010-2011.

[8] Chai Keong Toh Elizabethm Royer, "A review of current routing protocols for ad hoc mobile wireless networks," In Personal Communications, IEEE, vol. 6, no. 2, pp. 46-55, 1999.

[9] Sunil Taneja and Divya Sharma Ashwani Kush, "Energy Efficient Routing for MANET," International Conference on Methods and Models in Computer Science (ICM2CS), pp. 112-116, 2010.

[10] David A. Maltz David B. Johnson, "Dynamic source routing in ad hoc wireless networks," In Mobile Computing, Kluwer Academic Publishers, 1996.

[11] Amir Masoud Rahmani, Ehsan Aminian Ebrahim Mahdipour, "Performance Evaluation of Destination Sequenced Distance-Vector (DSDV)Routing Procol," International Conference on Future Networks, pp. 186 - 190, 2009.

[12] P. Macker D. Park, "Applicability of the temporally-ordered routing algorithm for use in mobile tactical networks," in Military Communications Conference, 1998. MILCOM 98. Proceedings.,IEEE, 1998. 